\section{Síndromes hipertensivas da gestação e repercussões perinatais}

\section{Hypertensive syndromes during pregnancy and perinatal outcomes}

Cristiane Alves de Oliveira 1

Carla Pereira Lins 2

Renato Augusto Moreira de Sá 3

Hermógenes Chaves Netto 4

Rita Guerios Bornia 5

Nancy Ribeiro da Silva 6

Joffre Amim Junior 7

\author{
1,2,6 Setor de Pesquisa e Extensão. Maternidade Escola. \\ Universidade Federal do Rio de Janeiro. \\ Rua das Laranjeiras, 180. Laranjeiras. Rio de Janeiro, RJ. Brasil. \\ CEP: 22.240-000 E-mail: crisalves.oliveira@openlink.com.br \\ 3 Setor de Obstetrícia. Universidade Federal Fluminense. Niterói, \\ RJ, Brasil \\ 4,5,7 Setor de Obstetrícia. Universidade Federal do Rio de Janeiro, \\ RJ, Brasil
}

\section{Resumo}

Objetivos: avaliar repercussões perinatais nas síndromes hipertensivas em gestações.

Métodos: estudo observacional e retrospectivo, realizado em hospital terciário, entre janeiro de 1996 e outubro de 2003. Um total de 12.272 gestações preencheu critérios de inclusão. Dois tipos de hipertensão foram considerados: hipertensão gestacional $(H G)$ e hipertensão arterial crônica (HAC). Variáveis estudadas: fetos pequenos para idade gestacional (PIG), Apgar baixo no $1^{\circ}$ e $5^{\circ}$ minutos, infecção neonatal, síndrome de aspiração meconial (SAM), prematuridade, síndrome de angústia respiratória (SAR).

Resultados: 1259 (10,26\%) gestantes tinham hipertensão; 344 (2,80\%) foram classificadas como $H G$, 915 (7,45\%) como HAC, havendo 11.013 (89,74\%) gestantes normotensas. HG constituiu risco elevado para: PIG, Apgar baixo no $1^{\circ}$ e $5^{\circ}$ minutos, infecção neonatal e prematuridade, mas não para SAM e SAR. HAC constituiu risco elevado para: PIG, Apgar baixo no $1^{\circ}$ minuto, SAM, prematuridade e SAR, mas não para Apgar baixo no $5^{\circ}$ minuto e infecção neonatal. Quando comparamos os riscos relativos dos grupos de HAC e $H G$, houve maior risco de prematuridade no grupo de HAC.

Conclusões: dados sugerem que tanto HAC quanto $H G$ aumentaram risco para PIG, Apgar baixo no $1^{\circ}$ e $5^{\circ}$ minutos, infecção neonatal, SAM, prematuridade e SAR. HAC apresentou maior risco relativo para prematuridade.

Palavras-chave Assistência perinatal; Hipertensão, Pré-eclâmpsia, Gravidez de alto-risco 


\section{Introdução}

A hipertensão é a complicação clínica mais comum da gestação, ocorrendo em 10 a $22 \%$ das gestações. ${ }^{1-3}$

O termo hipertensão induzida pela gestação refere-se ao aumento da pressão arterial que se manifesta apenas na gravidez. 4

As síndromes hipertensivas que acometem a mulher grávida são habitualmente classificadas em "hipertensão gestacional" (pressão arterial $\geq 140$ x 90 mmHg diagnosticada pela primeira vez na gestação, ausência de proteinúria, retorno aos níveis tencionais até 12 semanas após o parto), "pré-eclâmpsia" (pressão arterial $\geq 140$ x $90 \mathrm{mmHg}$ diagnosticada após 20 semanas de gestação associada à proteinúria $\geq 300$ mg/24 horas), "hipertensão arterial crônica" (pressão arterial $\geq 140 \times 90 \mathrm{mmHg}$ diagnosticada antes da gestação ou antes de 20 semanas de gestação não-atribuída à doença trofoblástica gestacional ou pressão arterial $\geq 140$ x $90 \mathrm{mmHg}$ diagnosticada após 20 semanas de gestação que persiste após 12 semanas de pós-parto), eclâmpsia (presença de convulsão, que não pode ser atribuída a outras causas, em mulheres com pré-eclâmpsia) e "préeclâmpsia sobreposta" (surgimento de proteinúria $\geq 300 \mathrm{mg} / 24$ horas em paciente hipertensa que não apresentava proteinúria antes de 20 semanas de gestação ou aumento importante da proteinúria, da pressão arterial ou plaquetas $<100.000 / \mathrm{mm}^{3} \mathrm{em}$ gestante hipertensa com proteinúria presente antes de 20 semanas de gestação). 4

As síndromes hipertensivas na gestação diferem quanto à prevalência, gravidade e efeitos sobre o feto. 5

A prevalência de hipertensão gestacional em pacientes nulíparas é 6 a $17 \%$, sendo 2 a $4 \%$ em pacientes multíparas. ${ }^{1}$ Em $20-50 \%$ das pacientes com hipertensão gestacional há progressão para $\mathrm{PE}$, ou seja, há desenvolvimento de proteinúria, podendo a mesma se desenvolver antes ou após o parto. ${ }^{6}$

A prevalência de pré-eclâmpsia (PE) é normalmente descrita como 5 a $8 \%$, apresentando amplas variações na literatura. Em gestação gemelar a prevalência de PE é de $14 \%$, podendo chegar a $40 \%$ em pacientes com PE prévia. ${ }^{3,4,7}$

A hipertensão arterial crônica (HAC) acomete em torno de $5 \%$ das gestações, sendo considerada como fator de risco para a PE. A taxa de PE sobreposta em pacientes hipertensas crônicas é 15 a $25 \% .8,9$

A maioria dos casos de hipertensão gestacional/pré-eclâmpsia leve se desenvolve próximo ao termo e apresenta taxas de mortalidade e morbidade perinatais similares às de pacientes normotensas. $1,4,5$

A hipertensão gestacional/pré-eclâmpsia grave é potencialmente a que apresenta pior prognóstico materno-fetal. Conceptos de mães com PE ou PE sobreposta têm maiores riscos de prematuridade, ocorrência de partos de fetos pequenos para a idade gestacional (PIG), necessidade de Unidade de Tratamento Intensivo (UTI) neonatal, necessidade de suporte ventilatório e maior incidência de mortalidade perinatal, quando comparados aos conceptos de mães normotensas. ${ }^{4}$ Ray et al., 5 estudaram os resultados perinatais nas diferentes síndromes hipertensivas, observando maiores riscos de prematuridade e baixo peso ao nascimento nas pacientes com PE e PE sobreposta quando comparadas à hipertensão gestacional. A ocorrência de doenças associadas à prematuridade (SAR, hemorragia intraventricular e enterocolite necrotizante), necessidade de ventilação assistida, admissão em UTI neonatal e taxa de mortalidade perinatal foram maiores nas pacientes com PE e com PE sobreposta quando comparadas às pacientes com hipertensão gestacional e HAC isolada.

A piora do prognóstico materno-fetal está diretamente relacionada à gravidade da hipertensão gestacional/pré-eclâmpsia. Hauth et al.,10 compararam os resultados perinatais em gestantes normotensas com gestantes que desenvolveram hipertensão na gestação e observaram piores resultados no grupo de hipertensas, principalmente nos casos graves. As variáveis estudadas foram: parto com idade gestacional inferior a 34 semanas de gestação, crescimento intra-uterino restrito (CIUR), admissão em UTI neonatal, peso ao nascimento inferior a $2500 \mathrm{~g}$, síndrome de angústia respiratória (SAR) e necessidade de ventilação assistida.

Em pacientes com HAC, a presença de PE sobreposta é a maior responsável pela piora da morbimortalidade perinatal, havendo também maiores taxas de resultados perinatais adversos em pacientes com HAC grave e não-controlada. ${ }^{4,10,11}$ Rey et al., ${ }^{11}$ compararam os resultados perinatais de pacientes com HAC com os resultados de pacientes normotensas, observando maiores taxas de mortalidade perinatal $(45 / 1000 \times 12 / 1000, p<0,001)$, parto prematuro $(34,4 \% \times 15,0 \%, p<0,01)$ e feto pequeno para a idade gestacional $(15,5 \% \times 6,3 \%, p<0,01)$ nas pa cientes hipertensas crônicas. Também foram mais freqüentes no grupo com HAC o baixo peso ao nascimento e baixos índices de Apgar no $7^{\circ}$ e no $10^{\circ}$ minutos. 11

Sibai et al., ${ }^{12}$ observaram maior risco de prematuridade $(56 \%$ × $25 \%, p<0,001)$ e maior mortalidade perinatal $(8 \% \times 4 \%, p=0,02)$ nas pacientes com $\mathrm{PE}$ sobreposta quando comparadas às pacientes com 


\section{HAC isolada. 12}

Com o objetivo de estudar o impacto das síndromes hipertensivas nos resultados perinatais, foram avaliadas retrospectivamente gestantes que tiveram parto na Maternidade Escola da Universidade Federal do Rio de Janeiro.

\section{Métodos}

Estudo do tipo observacional e retrospectivo, onde foram avaliadas retrospectivamente todas as pacientes que tiveram parto no período entre $1^{\circ} \mathrm{de}$ janeiro de 1996 e 31 de outubro de 2003 na Maternidade Escola da Universidade Federal do Rio de Janeiro (UFRJ), Rio de Janeiro, Brasil.

As informações das gestações foram obtidas através de pesquisa na Base de Dados da Maternidade o Sistema Informático Perinatal do Centro Latino Americano de Perinatologia (SIP/CLAP). Os dados analisados foram registrados em formulário próprio do Sistema por médicos da instituição na ocasião da alta hospitalar de mãe e neonato após o parto. As informações registradas foram provenientes da revisão da história clínica e exames realizados pelas pacientes durante o pré-natal e internação hospitalar (anotados no prontuário).

O critério de inclusão foi parto de concepto com idade gestacional superior a 20 semanas e gestação única.

Definiu-se como hipertensão arterial a presença de pressão arterial maior ou igual a 140 x $90 \mathrm{mmHg}$ em pelo menos duas ocasiões, com intervalo de quatro horas ou mais. A pressão arterial da gestante foi aferida após repouso mínimo de 10 minutos, com a gestante sentada e antebraço direito posicionado horizontalmente ao nível do coração, utilizando-se esfignomanômetro de coluna de mercúrio. Foram utilizados o primeiro e o quinto ruídos de Korotkoff como limites superior e inferior da pressão arterial. O American College of Obstetricians and Gynecologists (ACOG) considera a fase $\mathrm{V}$ como tendo maior precisão na avaliação da pressão diastólica. As aferições da pressão arterial foram feitas durante as consultas de pré-natal e na internação hospitalar.

As gestantes foram divididas em três grupos: Grupo 1: gestantes portadoras de hipertensão desenvolvida na gestação (HG) (gestantes que desenvolveram hipertensão a partir de 20 semanas de gestação, com e sem proteinúria); Grupo 2: gestantes portadoras de hipertensão arterial crônica (hipertensão arterial diagnosticada previamente à gestação ou diagnosticada antes de 20 semanas de gestação) e Grupo 3: gestantes normotensas (grupo controle).
Foram realizadas as análises entre o Grupo 1 (HG) e o Grupo 3 (grupo controle), entre os Grupo 2 (HAC) e Grupo 3 (grupo controle), e finalmente foram comparados os riscos relativos obtidos para os Grupo 1 (HG) e o Grupo 2 (HAC).

As variáveis de estudo foram: a) parto de fetos classificados como pequenos para a idade gestacional (PIG); b) baixo índice de Apgar no $1^{\circ}$ e no $5^{\circ}$ minutos após o nascimento; c) infecção neonatal; d) síndrome de aspiração de mecônio (SAM); e) prematuridade; e f) síndrome de angústia respiratória (SAR).

Considerou-se como "PIG": peso ao nascimento abaixo do $10^{\circ}$ percentil para a idade gestacional; "índices de Apgar baixos": quando menores que 7; "infecção neonatal": presença de clínica e exames laboratoriais sugestivos de quadro infeccioso no neonato; "SAM": visualização de mecônio na traquéia do concepto associada à clínica de desconforto respiratório; "prematuridade": nascimento antes de 37 semanas de gestação; e "SAR": necessidade de ventilação assistida e/ou suplementação de oxigênio nas primeiras 24 horas após o parto.

Calculou-se o risco relativo (RR) e seus limites no intervalo de confiança a $95 \%$. Para avaliar a associação entre as variáveis estudadas utilizou-se o teste do Qui-quadrado, considerado como significativo quando $p<0,05$.

\section{Resultados}

Foram selecionadas para o estudo 12.272 gestantes, das quais $1259(10,26 \%)$ apresentaram pressão arterial igual ou superior a $140 / 90 \mathrm{mmHg}$. No Grupo 1 (HG) e no Grupo 2 (HAC) foram incluídas 344

Tabela 1

Comparação entre Grupo 1 (hipertensão gestacional) e Grupo 3 (grupo controle) quanto ao risco de desenvolvimento de complicações perinatais.

\begin{tabular}{lrrrrr}
\hline & RR & \multicolumn{2}{c}{ IC95\% } & \multicolumn{1}{c}{$\chi^{2}$} & \multicolumn{1}{c}{$\boldsymbol{p}^{*}$} \\
\hline PIG & 2,10 & 1,77 & 2,48 & 73,948 & $<0,001$ \\
Apgar $1^{\circ}$ minuto & 1,26 & 1,05 & 1,52 & 6,198 & 0,012 \\
Apgar $5^{\circ}$ minuto & 1,45 & 1,00 & 2,09 & 3,986 & 0,045 \\
Infecção Neonatal & 3,12 & 1,74 & 5,59 & 16,111 & $<0,001$ \\
SAM & 1,49 & 0,90 & 2,46 & 2,498 & 0,113 \\
Prematuridade & 1,79 & 1,55 & 2,07 & 59,850 & $<0,001$ \\
SAR & 1,39 & 0,89 & 2,18 & 2,154 & 0,140 \\
\hline
\end{tabular}

RR=Risco relativo; IC95\%=Intervalo de Confiança $95 \% ; \chi 2=$ teste do Quiquadrado; $p=\mathrm{p}$ valor; $\mathrm{PIG}=$ Pequeno para a idade gestacional; $\mathrm{SAM}=$ Síndrome de Aspiração de Mecônio; SAR=Síndrome de Angústia Respiratória; $p$ * considerado como significativo quando $<0,05$. 
gestantes $(2,80 \%)$ e 915 gestantes $(7,45 \%)$ respectivamente. No Grupo 3 (grupo controle) foram incluídas 11.013 gestantes $(89,74 \%)$.

Quando comparados os Grupos 1 e 2 isoladamente com o Grupo 3 (grupo controle), o Grupo 1 (HG) apresentou risco aumentado para: PIG $(\mathrm{RR}=2,10, p<<0,001)$, Apgar baixo no $1^{\circ}$ minuto ( $\mathrm{RR}=1,26, p=0,012)$, Apgar baixo no $5^{\circ}$ minuto $(\mathrm{RR}=1,45, p=0,045)$, infecção neonatal $(\mathrm{RR}=3,12$, $p<<0,001)$ e prematuridade ( $R R=1,79, p<<0,001)$. Entretanto, a presença de HG não se relacionou ao maior risco para $\mathrm{SAM}(\mathrm{RR}=1,49, p=0,113)$ e $\mathrm{SAR}$ $(\mathrm{RR}=1,39, p=0,142)$ (Tabela 1$)$.

\section{Tabela 2}

Comparação entre Grupo 2 (hipertensão arterial crônica) e Grupo 3 (grupo controle) quanto ao risco de desenvolvimento de complicações perinatais.

\begin{tabular}{lrrrrr}
\hline & RR & \multicolumn{2}{c}{ IC95\% } & $\chi^{2}$ & \multicolumn{1}{c}{$\boldsymbol{p}^{*}$} \\
\hline PIG & 1,87 & 1,45 & 2,48 & 18,225 & $<0,001$ \\
Apgar $1^{\circ}$ minuto & 1,65 & 1,28 & 2,12 & 14,712 & $<0,001$ \\
Apgar $5^{\circ}$ minuto & 1,49 & 0,84 & 2,64 & 1,923 & 0,165 \\
Infecção neonatal & 0,59 & 0,08 & 4,27 & 0,275 & 0,599 \\
SAM & 2,33 & 1,24 & 4,40 & 7,220 & 0,007 \\
Prematuridade & 2,45 & 2,03 & 2,96 & 80,337 & $<0,001$ \\
SAR & 1,94 & 1,06 & 3,54 & 4,848 & 0,027 \\
\hline
\end{tabular}

$\mathrm{RR}=\mathrm{Risco}$ relativo; IC95\%=Intervalo de Confiança $95 \% ; \chi 2=$ teste do Quiquadrado; $p=\mathrm{p}$ valor; $\mathrm{PIG}=$ Pequeno para a idade gestacional; SAM=Síndrome de Aspiração de Mecônio; SAR=Síndrome de Angústia Respiratória; $p$ * considerado como significativo quando <0,05.

Tabela 3

Comparação dos riscos relativos para desenvolvimento de complicações perinatais referentes ao Grupo 2 (hipertensão arterial crônica) e ao Grupo 1 (hipertensão gestacional).

\begin{tabular}{lccccc}
\hline & RR & \multicolumn{2}{c}{ IC95\% } & $\chi^{2}$ & $\boldsymbol{p}^{*}$ \\
\hline PIG & 0,88 & 0,65 & 1,21 & 0,545 & 0,460 \\
Apgar $1^{\circ}$ minuto & 1,30 & 0,96 & 1,76 & 2,981 & 0,084 \\
Apgar $5^{\circ}$ minuto & 1,02 & 0,53 & 1,98 & 0,007 & 0,930 \\
Infecção Neonatal & 0,19 & 0,02 & 1,43 & 3,262 & 0,700 \\
SAM & 1,56 & 0,72 & 3,38 & 1,311 & 0,252 \\
Prematuridade & 1,36 & 1,09 & 1,71 & 7,359 & 0,006 \\
SAR & 1,39 & 0,67 & 2,85 & 0,822 & 0,364 \\
\hline
\end{tabular}

RR=Risco relativo; IC95\%=Intervalo de confiança $95 \% ; \chi 2=$ teste do Quiquadrado; $p=p$ valor; $\mathrm{PIG}=$ Pequeno para a idade gestacional; $\mathrm{SAM}=$ Síndrome de Aspiração de Mecônio; SAR = Síndrome de Angústia Respiratória; $p$ * considerado como significativo quando $<0,05$.
O Grupo 2 (HAC) apresentou risco relativo aumentado para: PIG ( $\mathrm{RR}=1,87, p<0,001)$, Apgar baixo no $1^{\circ}$ minuto $(\mathrm{RR}=1,65, p<0,001)$, para $\mathrm{SAM}$ $(\mathrm{RR}=2,33, p=0,007)$, prematuridade $(\mathrm{RR}=2,45$, $p<0,001)$ e $\mathrm{SAR}(\mathrm{RR}=1,94, p=0,027)$, mas não para Apgar baixo no $5^{\circ}$ minuto $(\mathrm{RR}=1,49, p=0,165) \mathrm{e}$ infecção neonatal ( $R R=0,59, p=0,599)$ (Tabela 2).

Quando comparados os riscos relativos encontrados para as pacientes com HAC com aqueles das pacientes com HG, verificou-se que não houve diferença estatisticamente significativa entre os dois grupos quanto ao risco relativo para ocorrência das seguintes variáveis: PIG ( $\mathrm{RR}=0,88, p=0,460)$, Apgar baixo no $1^{\circ}$ minuto $(\mathrm{RR}=1,30, p=0,084)$, Apgar baixo no $5^{\circ}$ minuto $(\mathrm{RR}=1,02, p=0,930)$, infecção neonatal $(\mathrm{RR}=0,19, p=0,070), \mathrm{SAM} \quad(\mathrm{RR}=1,56$, $p=0,252)$ e $\mathrm{SAR}(\mathrm{RR}=1,39, p=0,364)$. Entretanto, houve maior risco relativo de prematuridade no grupo de hipertensão arterial crônica $(\mathrm{R}=1,36$, $p=0,006$ ) quando comparado com o risco relativo do grupo com HG (Tabela 3).

\section{Discussão}

A hipertensão está associada a um aumento significativo de complicações perinatais. O impacto das síndromes hipertensivas na gestação tem sido estudado em vários trabalhos, havendo resultados variados na literatura.

O grande tamanho amostral obtido da base de dados pode prover uma boa visão da população estudada.

A prevalência de $10,26 \%$ de pacientes hipertensas na população estudada está de acordo com a literatura sobre o assunto, que descreve esta prevalência entre 10 e $22 \%$. Devido à inconsistência dos dados obtidos através de pesquisa na Base de Dados da Maternidade Escola da UFRJ, não foi possível classificar os diferentes tipos de hipertensão induzidas pela gestação (de acordo com a presença ou não de proteinúria), sendo a análise deste estudo limitada ao grupo de pacientes com hipertensão desenvolvida na gestação (HG), independente da presença de proteinúria e ao grupo de pacientes com diagnóstico de hipertensão arterial crônica (HAC).

A HAC foi mais prevalente que a HG na população estudada (respectivamente $7,45 \%$ e $2,80 \%$ da população total). Dentre as pacientes hipertensas $27,32 \%$ foram diagnosticadas como $\mathrm{HG}$ e $72,67 \%$ como HAC. Ao contrário do presente estudo, Jain, 13 tendo estudado retrospectivamente 109.428 gestantes, encontrou maior prevalência de $\mathrm{HG}$ em relação à HAC. Embora na maior parte da literatura as 
prevalências de pré-eclâmpsia e de hipertensão gestacional sejam normalmente maiores que a prevalência de HAC, um resultado semelhante ao do presente estudo foi encontrado por Gaio et al.,14 ao estudar 4892 gestantes na Universidade do Rio Grande do Sul, encontrando 4,0\% de gestantes com HAC, $0,7 \%$ com diagnóstico de hipertensão gestacional e 2,3\% com pré-eclâmpsia/eclâmpsia.

A presença de diagnóstico de hipertensão arterial aumentou o risco para ocorrência de todas as variáveis estudadas, resultado também encontrado pelos autores acima citados.

Embora a HAC não tenha aumentado significativamente o risco de ocorrência de baixo índice de Apgar no $5^{\circ}$ minuto e de infecção perinatal, e a HG não tenha gerado aumento significativo do risco de SAM e SAR nos recém-natos quando comparados aos do grupo controle (pacientes normotensas), não houve diferença estatisticamente significativa entre esses resultados perinatais quando os riscos relativos dos grupos com HAC e HG foram comparados entre si, com exceção da prematuridade, tendo sido encontrado maior risco relativo de prematuridade nas pacientes hipertensas crônicas quando comparadas às gestantes com hipertensão desenvolvida na gestação. Ray et a.l,5 estudaram 1948 gestantes hipertensas e compararam pacientes com hipertensão gestacional com gestantes com as demais síndromes hipertensivas: os recém-natos de gestantes com HAC

\section{Referências}

1. Sibai BM. Diagnosis and management of gestational hypertension and preeclampsia. Obstet Gynecol 2003; 102: 18192.

2. Livingston JC, Sibai BM. Chronic hypertension in pregnancy. Obstet Gynecol Clin North Am 2001; 28: 447-63.

3. ACOG (American College of Obstetricians and Gynecologists). Committee on Practice BulletinsObstetrics. Diagnosis and management of preeclampsia and eclampsia. Obstet Gynecol 2002; 33: 159-67.

4. Cunningham FG, Gant NF, Leveno KJ, Gilstrap III LC, Hauth JC, Wenstrom KD. Hypertensive disorders in pregnancy. In: Williams JW, editor. Williams Obstetrics. Houston: Mc Graw-Hill; 2001. p. 567-617.

5. Ray JG, Burrows RF, Burrows EA, Vermeulen MJ. MOS HIP: Mc Master outcome study of hypertension in pregnancy. Early Hum Dev 2001; 64: 129-143

6. Buchbinder A, Sibai BM, Caritis S, MacPherson C, Hauth J, Lindheimer MD, Klebanoff M, VanDorsten P, Landon M, Paul R, Miodovnik M, Meis P, Thurnau G. Adverse perinatal outcome are significantly higher in severe gestational hypertension than in mild preeclampsia. Am J Obstet Gynecol 2002; 186: 66-71. não apresentaram maior risco de prematuridade (parto antes de 37 semanas de gestação) quando comparados aos de mães com hipertensão gestacional; houve aumento do risco de prematuridade nas pacientes com pré-eclâmpsia e com préeclâmpsia sobreposta quando comparadas com o grupo com hipertensão gestacional.

De acordo com os resultados encontrados no presente estudo, a HG não mostrou associação com piores resultados perinatais quando comparada a HAC. Tal resultado não está de acordo com a maioria da literatura disponível. Uma causa provável dessa diferença é o fato de não terem sido diferenciados os casos de HG leve daqueles com critérios de gravidade, já que são os casos graves de hipertensão gestacional que cursam com pior repercussão fetal, enquanto que os casos leves têm desfechos semelhantes aos de gestantes normotensas. 1

O presente estudo permitiu concluir que as síndromes hipertensivas na gestação, tanto a hipertensão arterial crônica quanto a hipertensão gestacional, aumentaram o risco para desfecho perinatal desfavorável (PIG, Apgar baixo no $1^{\circ}$ e $5^{\circ}$ minutos, infecção neonatal, SAM, prematuridade e SAR). Quando comparados os risco relativo para desfecho perinatal desfavorável entre HAC e HG, a hipertensão arterial crônica apresentou risco relativo de prematuridade significativamente maior.
7. Hnat MD, Sibai BM, Caritis S, Hauth J, Lindheimer MD, MacPherson C, VanDorsten P, Landon M, Miodovnik M, Paul R, Meis P, Thurnau G, Dombrowski M. Perinatal outcome in women with recurrent preeclampsia compared with women who develop preeclampsia as nulliparas. Am J Obstet Gynecol 2002; 186: 422-6.

8. Netto HC. Obstetrícia básica. In: Vasconcelos MJA, editor. Pré-eclâmpsia: doença hipertensiva vascular crônica. São Paulo: Atheneu; 2004. p. 247-57, 365-70.

9. ACOG (American College of Obstetricians and Gynecologists). Committee on Practice BulletinsObstetrics. Chronic hypertension in pregnancy. Obstet Gynecol 2001; 29: 177-85.

10. Hauth JC, Ewell MG, Levine RJ, Esterlitz JR, Sibai BM, Curet LB, Catalano PM, Morris CD. Pregnancy outcomes in health nulliparas who developed hypertension. Obstet Gynecol 2000; 95: 24-8.

11. Rey E, Couturier A. The prognosis of pregnancy in women with chronic hypertension. Am J Obstet Gynecol 1994; 171: 410-16. 
12. Sibai BM, Lindheimer MD, Hauth J, Caritis S, VanDorsten P, Klebanoff M, MacPherson C, Landon M, Miodovnik M, Paul R, Meis P, Dombrowski M. Risk factors for preeclampsia, abruptio placentae, and adverse neonatal outcomes among women with chronic hypertension. N Engl J Med 1998; 339: 667-71.

13. Jain L. Effect of pregnancy-induced and chronic hypertension on pregnancy outcome. J Perinatol 1997; 17: 425-7.
14. Gaio DS, Schmidt MI, Duncan BB, Nucci LB, Matos MC, Branchtein L. Hypertensive disorders in pregnancy: frequency and associated factors in a cohort of Brazilian women. Hypertens Pregnancy 2001; 20: 269-81.

Recebido em 23 de setembro de 2004

Versão final apresentada em 31 de janeiro de 2006

Aprovado em 3 de fevereiro de 2006 